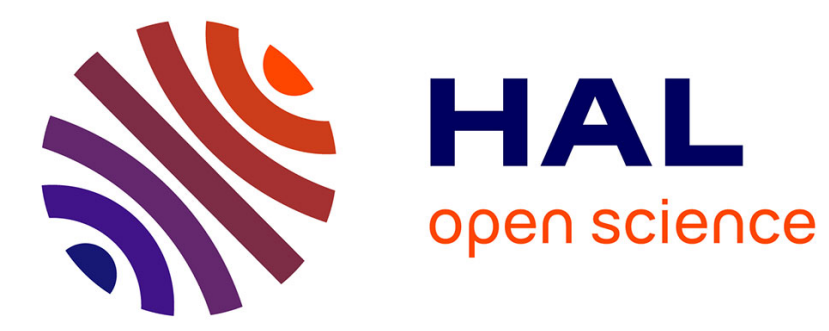

\title{
On the notion of thermophoretic velocity
}

Eric Bringuier

\section{To cite this version:}

Eric Bringuier. On the notion of thermophoretic velocity. Philosophical Magazine, 2007, 87 (06), pp.873-883. 10.1080/14786430601003841 . hal-00513776

\section{HAL Id: hal-00513776 \\ https://hal.science/hal-00513776}

Submitted on 1 Sep 2010

HAL is a multi-disciplinary open access archive for the deposit and dissemination of scientific research documents, whether they are published or not. The documents may come from teaching and research institutions in France or abroad, or from public or private research centers.
L'archive ouverte pluridisciplinaire HAL, est destinée au dépôt et à la diffusion de documents scientifiques de niveau recherche, publiés ou non, émanant des établissements d'enseignement et de recherche français ou étrangers, des laboratoires publics ou privés. 


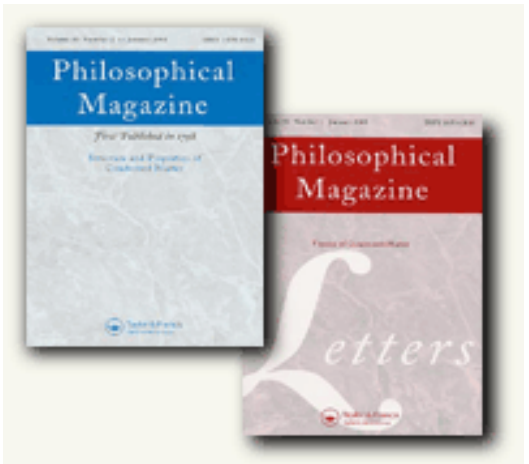

\section{On the notion of thermophoretic velocity}

\begin{tabular}{|r|l|}
\hline Journal: & Philosophical Magazine \& Philosophical Magazine Letters \\
\hline Manuscript ID: & TPHM-06-Apr-0085.R1 \\
\hline Journal Selection: & Philosophical Magazine \\
\hline Date Submitted by the \\
Author: & 08-Sep-2006 \\
\hline Complete List of Authors: & $\begin{array}{l}\text { Bringuier, Eric; Université Denis Diderot (Paris 7), Matériaux et } \\
\text { Phénomènes Quantiques (UMR 7162 CNRS) }\end{array}$ \\
\hline Keywords: & non-equilibrium phenomena, soft matter, Soret effect \\
\hline Keywords (user supplied): & thermophoresis, thermal diffusion \\
\hline &
\end{tabular}

\section{S ScholaroNE" \\ Manuscript Central}


Telephone 00.33.1.44274200

Fax 00.33.1.44273882

E-mail address erb@ccr.jussieu.fr

\author{
E. BRINGUIER
}

Matériaux et Phénomènes Quantiques (UMR 7162 CNRS)

Université Denis Diderot (Paris 7), case 7021

2 place Jussieu, 75251 Paris Cedex 05, France

\begin{abstract}
The phenomenology of thermal diffusion of a particle in a solvent involves the Soret coefficient of the particle/solvent mixture. It is usually considered that the migration velocity of the particle in the solvent is proportional to the Soret coefficient. I show here that this view is wrong because ordinary diffusion contributes to migration if the solvent is not homogeneous. I examine several examples $(\mathrm{NaCl}$ in water, polystyrene in ethylbenzene, maghemite nanoparticules) to show that this contribution can be strong enough to change the sign of the migration velocity and vitiate the interpretation of experimental data, the measurement of the Soret coefficient, the comparison of experiment with theory, and interexperimental comparisons.
\end{abstract}

Keywords: thermophoresis; thermal diffusion; Soret effect

PACS: 66.10.Cb, 51.10.+y

\title{
§ 1. Introduction
}

In a binary mixture, thermodiffusion, also called thermal diffusion or the Ludwig-Soret effect, is the partial demixtion induced by the application of a temperature gradient. It is the relative transport of a species, say 1, with respect to the other one, 2. In this paper, the species 1, henceforth called the solute, is taken to be very dilute among a liquid or gaseous medium made up of molecules 2, called the solvent. The Ludwig-Soret effect has been discovered 150 years ago in liquids [1,2] and some 90 years ago in gases [3]. The variety of mixtures found in soft matter science has recently generated a number of studies $[4,5]$. Thermodiffusion of $\mathrm{NaCl}$ plays a role in the thermohaline circulation, a key ingredient in global climate warming models [6], and thermodiffusion of DNA could play a major role in the origin of life [7]. 
The local state of a binary mixture is defined by the temperature $T$, the pressure $p$ and the mole fractions $x_{1}=n_{1} / n$ and $x_{2}=1-x_{1}$ of the components $\left(n_{1}\right.$ and $n_{2}$ are the number densities of species 1 and 2, in $\mathrm{m}^{-3}$ in SI unit, and $n=n_{1}+n_{2}$ is the total density). Starting from a homogeneous mixture where $x_{1}$ and $x_{2}$ are independent of position, the gradient of temperature causes a transport of particles 1 relative to 2, directed along or against $\nabla T$ in an isotropic medium. Transport is phenomenologically described by the number current density $j_{1}$ (in $\mathrm{m}^{-2} \cdot \mathrm{s}^{-1}$ in SI unit). As the ensuing inhomogeneity in the solute density entails a diffusion contribution to the current, $\boldsymbol{j}_{1}$ is expressed as

$$
j_{1}=-D n\left(\nabla x_{1}+\sigma_{\mathrm{T}} x_{1} \nabla T\right),
$$

where $D$ is the particle diffusivity and $\sigma_{\mathrm{T}}$ is called the Soret coefficient [8-10]. We assume in equation (1) that the species 1 is very dilute in the solvent 2 , and this is why $j_{1}$ is linear in $x_{1}$. It is also assumed that the medium is in mechanical equilibrium, i.e. at a uniform pressure $p$. Otherwise, another contribution to $\boldsymbol{j}_{1}$ is present, which is proportional to $x_{1} \nabla p$ and called the barodiffusion current density $[8,10]$.

Other expressions for $\boldsymbol{j}_{1}$ can be found, where the mass fraction [11] or the volume fraction [12] is used instead of the mole fraction $x_{1}$. It is also possible to express $\boldsymbol{j}_{1}$ as a linear combination of $\nabla n_{1}$ and $\nabla T$, as

$$
j_{1}=-D \nabla n_{1}-D S^{*} n_{1} \nabla T,
$$

where

$$
S^{*}=\sigma_{\mathrm{T}}+\beta,
$$

and $\beta$ is the coefficient of thermal expansion of the solvent (see appendix A). We also introduce the dimensionless coefficient

$$
\alpha=T S^{*} .
$$

In water in standard conditions, $T \beta=0,075$; in a perfect gas, $T \beta=1$. The difference between $S^{*}$ and $\sigma_{\mathrm{T}}$ can be important in a gas if $|\alpha|$ is close to unity. It is often ignored in a liquid.

Thermodiffusion has been studied independently in distinct areas. When the solute 1 is a macromolecule, it is usual to call thermophoresis its motion in a temperature gradient, in analogy with electrophoresis, which is the motion of a charged solute in an electric field. Generally speaking, the effect for small molecules 1, or for gaseous or liquid mixtures having comparable proportions of 1 and 2, is called thermodiffusion, while for suspensions of particles in gases or in liquids, it is called thermophoresis [13-19]. Then, $D S^{*}$ is called the thermophoretic mobility, in analogy with the electrophoretic mobility, and accordingly $-D S^{*} \nabla T$ is called the thermophoretic velocity. In what follows, I consider a dilute suspension of particles 1 in a solvent 2, and I drop the index 1 .

The point I want to make in this paper is that the wording thermophoretic velocity is fallacious. It is drawn from the usual interpretation of the coefficients in a migration-diffusion current equation, also called advection-diffusion or convection-diffusion equation, formally similar to equation (2). Unfortunately, that interpretation is not valid in an inhomogeneous 
medium, as occurs here because of the temperature non-uniformity. Stated otherwise, I want to show that a particle does not migrate at the velocity $-D S^{*} \nabla T$ through the solvent. Likewise, the notion of an effective force $-\alpha \nabla(k T)$, sometimes termed "thermal force", propelling the particle against $(\alpha>0)$ or along $(\alpha<0)$ the temperature gradient with a mobility $D / k T$ inferred from the Nernst-Einstein relation, is misleading. It is drawn from viewing equation (2) as a forced diffusion equation [10]. The incorrectness of the identification of the migration velocity with $-D S^{*} \nabla T$ is shown in $\S 2$, which is based upon the phenomenological current equation only, with no appeal to any underlying theory or assumption. In $\S 3$, I pick up three applications to the phenomenology of thermophoresis and the experimental determination of $S^{*}$. Conclusions are gathered in $\S 4$.

\section{$\S 2$. The migration velocity}

\section{$\S 2.1$. Derivation of the migration velocity from the current equation}

Consider a statistical ensemble of particles in a vessel, and take one space dimension $x$ for simplicity. (In three dimensions the calculation is similar, with Gauss's divergence theorem replacing the integration by parts.) The vessel boundaries are $x=0$ and $x=L>0$. For definiteness, a hot source located at $x=0^{-}$and a cold source located at $x=L^{+}$establish the temperature gradient $d T / d x<0$ in the bulk (figure 1 ).

[Insert figure 1 about here]

Generally speaking, the migration, or drift, velocity $v_{\mathrm{d}}$ is the velocity of the centroid

$$
\langle x\rangle=\int x n(x, t) d x
$$

of the ensemble of particles, with $n$ being normalized to unity $\left(\int n(x, t) d x=1\right)$. To obtain $d\langle x\rangle / d t$, we use the equation of continuity,

$$
\frac{\partial n}{\partial t}+\frac{\partial j}{\partial x}=0,
$$

expressing the local conservation of particles. Multiply equation (6) by $x$ and integrate over $x$. Integration by parts and the vanishing of $x j(x, t)$ at the boundaries of the vessel entail

$$
d\langle x\rangle / d t=\int j(x, t) d x .
$$

The phenomenological current equation (2) may alternatively be rewritten as

$$
j=\left(-D S^{*} \frac{d T}{d x}+\frac{d D}{d x}\right) n-\frac{d(D n)}{d x} .
$$

The last term in (8) contributes $D(L) n(L, t)-D(0) n(0, t)$ to the right-hand side of (7). Assume that $n(x, t)$ vanishes at the vessel boundaries (the opposite case is examined in $\S 2.2$ ). Then,

$$
\frac{d\langle x\rangle}{d t}=\left\langle-D S^{*} \frac{d T}{d x}+\frac{d D}{d x}\right\rangle .
$$

Relation (9) shows that the sign of $d\langle x\rangle / d t$ is not determined by that of $S^{*}$ alone. The meaning of the second contribution to $d\langle x\rangle / d t$ in the right-hand side of (9) is easy to understand if we take, for the normalized density profile at time $t$, 
II The converse effect is also found, experimentally and theoretically, namely the change in the diffusivity due to an inhomogeneous drift velocity, in charge-carrier transport in a gas or a semiconductor subjected to a high electric field. Because the leading edge drifts at a slower velocity than the trailing edge, the carrier packet is squeezed along the direction of motion, and the longitudinal diffusivity is smaller than the transverse diffusivity. See [23] and references therein.

If the diffusivity is larger on the leading edge $x=x_{\mathrm{II}}$ than on the trailing edge $x=x_{\mathrm{I}}, d\langle x\rangle / d t$ will, algebraically speaking, exceed the average value of the "thermophoretic velocity" $-D S^{*}(d T / d x)$ over the particle distribution. No matter how large the packet is, its actual mean velocity $d\langle x\rangle / d t$ is altered by the differential spreading $d D / d x$, see figure 2. This is known in the study of stochastic processes [20-22] $\mathbb{\pi}$ and has been experimentally checked in an inhomogeneous colloidal solution [24]. Therefore, contrary to a widespread belief, the positivity of $S^{*}$ does not imply that the particles should migrate, on the average, to the cold side. Finally, in the limit $x_{\mathrm{I}} \rightarrow x \leftarrow x_{\mathrm{II}}$ of a $\delta$-peaked $n(x, t)$, the velocity is a function of $x$ only,

$$
v_{\mathrm{d}}(x)=-D S^{*} \frac{d T}{d x}+\frac{d D}{d x} .
$$

It is the mean velocity of a group of particles released at a definite position $x$ at time $t$. At later times, the centroid of the group moves at velocity $v_{\mathrm{d}}$ and the group spreads about the centroid. [Insert figure 2 about here]

The spreading of the group can be obtained from the phenomenological current equation (2). Calculations similar to (5)-(9) throw up

$$
\frac{d\left\langle x^{2}\right\rangle}{d t}-\frac{d\langle x\rangle^{2}}{d t}=2\langle D(x)\rangle+2\left\langle x v_{\mathrm{d}}(x)\right\rangle-2\langle x\rangle \frac{d\langle x\rangle}{d t} .
$$

If we consider a normalized $\delta$-peaked $n$ at time $t, n(x, t)$ is identical with $\delta(x-\langle x\rangle)$, so that (i) $\langle D(x)\rangle=D(\langle x\rangle)$ and (ii) the middle term in the right-hand side of (13) is $2\langle x\rangle\left\langle v_{\mathrm{d}}(x)\right\rangle$ and cancels the last term. As a result, the instantaneous rate of change of the variance in position is just $2 D(x)$ in one dimension. Therefore $D(x)$ retains its usual meaning of half the spreading rate of a packet initially located at position $x$, and may be thought of as the local diffusivity.

Assume now that the only dependence of $D$ on position is due to $T(x)$. Then, relation (12) can be rewritten as

$$
v_{\mathrm{d}}(x)=-D\left(S^{*}-\frac{d \ln D}{d T}\right) \frac{d T}{d x} .
$$

Thus, taking into account the temperature dependence of the diffusivity is tantamount to subtracting $d \ln D / d \ln T$ from $T S^{*}$ in determining the migration velocity caused by a temperature gradient. Section 3 will show on three specific examples that the correction to $S^{*}$ may be large enough to change its sign and/or flaw the comparison of experiment with theory and the consistency of different experimental methods aimed at measuring $S^{*}$. 
$\S 2.2$. The relationship between the migration velocity and the steady state

Consider a solute such that $S^{*}>0$ and $v_{\mathrm{d}}<0$ (we shall see in $\S 3.1$ that $\mathrm{NaCl}$ so behaves around $25^{\circ} \mathrm{C}$ ). Again, the vessel is the one depicted in figure 1 . How can we reconcile the direction of the migration velocity with the sign of $S^{*}$ in the steady state ? Then, $j=0$ and the density profile, denoted as $n_{\infty}(x)$, obeys

$$
\frac{d \ln n_{\infty}}{d x}=-S^{*} \frac{d T}{d x} .
$$

The equation governing the profile is formally identical with that of a sedimentation equilibrium due to a force $-\alpha d(k T) / d x>0$. Equation (15) shows that the positivity of $S^{*}$ is tantamount to the solute preferentially concentrating in the colder region $(x \rightarrow L)$ in the final state. Although the concept of a position-dependent particle velocity $v_{\mathrm{d}}(x)<0$ is still relevant in the steady state, it is insufficient because we are dealing with stochastic motion. In addition to the directed, or average, motion $v_{\mathrm{d}}(x)$, making the so-called orthokinetic contribution to $j$ [25], we have to take into account the fluctuations about the average, making the so-called perikinetic contribution to $j$. It is because of that contribution that, in the classic sedimentation equilibrium, the suspended particles reach a steady distribution although they everlastingly fall toward the bottom of the vessel. In the present problem, while the solute does drift toward $x=$ 0 , its higher diffusivity as $x \rightarrow 0$ entails a net backflow toward $x=L$. In this example, because the variation in $D(x)$ outweighs that in $n_{\infty}(x)$, the perikinetic contribution to $j$ disagrees in sign with Fick's law holding in a homogeneous medium where $D$ is independent of position $x$. Although the solute moves, on the average, to the hot side, much of it does not stay there because it diffuses farther on that side. The larger perikinetic outflow on the hot side causes the depletion near $x=0$, consistent with $S^{*}>0$. The profile $n_{\infty}(x)$ is determined by both the ortho- and perikinetic contributions to the flow, and this is why $v_{\mathrm{d}}$ may be negative while $-S^{*}(d T / d x)$ is positive.

In the steady state, the perikinetic contribution locally balances the orthokinetic one, $n v_{\mathrm{d}}(x)-d(D n) / d x=0$ (drift without current [24]). The integral balance reads

$$
\int_{0}^{L} v_{d}(x) n_{\infty}(x) d x+D(0) n_{\infty}(0)-D(L) n_{\infty}(L)=0 .
$$

Relation (16) replaces relation (9) which does not hold here as equation (15) prevents $n_{\infty}(x)$ from vanishing at both boundaries. Relation (16) may be thought of as a bulk migration $\left\langle v_{\mathrm{d}}\right\rangle<$ 0 being offset by a reflecting effect $[22,26]$ of the $x=0$ boundary ; the reflecting effect of the $x=L$ boundary enhances $\left\langle v_{\mathrm{d}}\right\rangle$, but it is weaker than at $x=0$.

\section{§3. Applications}

$\S 3.1 . \mathrm{NaCl}$ in water 
To show the practical implications of our relation (14), we now consider three examples of very different natures. The first one deals with the interpretation of $S^{*}$ in a solution first studied by Soret [2], namely the aqueous solution of $\mathrm{NaCl}$. The thermal diffusions of $\mathrm{Na}^{+}$and $\mathrm{Cl}^{-}$are coupled because the solution cannot significantly deviate from local electroneutrality, so that one $S^{*}$ coefficient is used for $\mathrm{NaCl}$ as a whole [8], although it is dissociated into ions. Similarly, the coupling of ordinary diffusions of $\mathrm{Na}^{+}$and $\mathrm{Cl}^{-}$is reflected in a single diffusivity $D$, the so-called ambipolar diffusivity [27]. At $0.5 \mathrm{~mol} \cdot \mathrm{L}^{-1}$, it is found [28] that thermodiffusion of $\mathrm{Na}^{+}$and $\mathrm{Cl}^{-}$has a $T S^{*}=+0.3$ at $25^{\circ} \mathrm{C}$, whose sign changes at $12{ }^{\circ} \mathrm{C}$. To determine the correction implied by relation (14), we have to determine the ambipolar diffusivity $D$ of $\mathrm{NaCl}$. It is given by $2 / D=1 / D_{+}+1 / D_{-}$[27] and the ionic diffusivities $D_{+}$and $D_{-}$can be obtained from the Nernst-Einstein relation $D_{ \pm}=k T \mu_{ \pm}$, where $\mu_{ \pm}$are the ionic mobilities. In water at $25{ }^{\circ} \mathrm{C}$ at standard pressure, it has been experimentally found that $d \ln \mu_{+} / d T=0.022 \mathrm{~K}^{-1}$ for $\mathrm{Na}^{+}$and $d \ln \mu_{\lrcorner} / d T=0.020 \mathrm{~K}^{-1}$ for $\mathrm{Cl}^{-}$[29]. We thus get $d \ln D / d \ln T=+7.3$, meaning that $D \propto T^{7.3}$, near $25^{\circ} \mathrm{C}$. This is a very strong temperature dependence. From relation (14), the correction to $T S^{*}$ is more than twenty times larger than $T S^{*}=+0.3$, with an opposite sign. This upsets the direction of the migration of the $\mathrm{Na}^{+}$and $\mathrm{Cl}^{-}$ions and the usual view held in studies of the thermohaline circulation in oceans [28]. The actual migration is towards the warmer temperatures. The incorrect interpretation is of no concern if the phenomenological equation (1) or (2) is correctly encoded in a computer programme. But, if physical reasoning is used, it entails qualitatively wrong conclusions.

More generally speaking, whenever $T S^{*}$ crosses the zero value, the diffusivity gradient changes the transition temperature from a "thermophobic" to a "thermophilic" behaviour. As a recent example involving proteins (top curve in figure 1 of [30]), $T S^{*}$ varies from -3.0 to +3.0 as $T$ spans the $280-310 \mathrm{~K}$ range, and vanishes at $290 \mathrm{~K}$. The measurement of $D(T)$ is not reported, but we may estimate $d \ln D / d \ln T=1-d \ln \eta / d \ln T$ from the Stokes-Einstein formula $D=k T / 6 \pi \eta R$ which is usually good for large-size spherical particles ( $\eta$ is the solvent viscosity and $R$ is the radius of the particle). We get $d \ln D / d \ln T=+8.2$ in water in standard conditions. (It is worthy of remark that even the temperature dependence $d \ln D / d \ln T=7.3$ of the diffusivity of the small-size $\mathrm{Na}^{+}$and $\mathrm{Cl}^{-}$ions computed above is well described by this formula.) Then, according to our relation (14), the protein migration is thermophilic throughout the $280-310 \mathrm{~K}$ range.

\section{$\S 3.2$. Thermal field-flow fractionation}

The second application of our relation (14) is the measurement of $S^{*}$ through thermal fieldflow fractionation, a high-resolution technique that uses thermophoresis to separate and characterise objects $[17,31]$. The solution flows between a hot wall and a cold wall whose 
temperature difference is $\Delta T$, along a direction parallel to the walls. What is measured is the shift $\Delta x$ of a solute injected at a definite position $x$, after a time $\Delta t$ has elapsed. From the thermophoretic velocity $U_{\mathrm{T}} \equiv \Delta x / \Delta t$ thus measured, a thermophoretic mobility is defined and computed as $D_{\mathrm{T}} \equiv-U_{\mathrm{T}} /(\Delta T / \Delta x)$. Then, it is compared to a theoretical prediction of that mobility. The experimentally defined quantity corresponds to our $-v_{\mathrm{d}} /(d T / d x)=D S^{*}-d D / d T$ according to relation (14), whereas the theoretical quantity is $D S^{*}$. Thus, the comparison between both quantities is flawed. The extent of the error depends on the values of the parameters. The values $T S^{*} \approx 45$ measured for polystyrene in ethylbenzene around $310 \mathrm{~K}$ (figure 9 in [31]) are not much affected by $d \ln D / d \ln T \approx 4.7$. However, this correction (10\%) is larger than the correction to the velocity profile of the flow between the hot and cold walls, which is also due to the temperature dependence of $\eta$ and is typically $4 \%$ [32].

\section{§ 3.3. Maghemite nanoparticules}

The values of $D$ and $S^{*}$ of $\gamma-\mathrm{Fe}_{2} \mathrm{O}_{3}$ nanoparticules have been measured by another experimental method, namely forced Rayleigh scattering [12,33] where a gradient of $T$ is that of a temperature grating created by a light-intensity grating. The phenomenology of the method rests upon equation (6) to describe the initial rise and the subsequent relaxation of $n$ following a light pulse. In equation (6), the current density is inputted with the pattern (2) in which $D$ and $S^{*}$, or $D$ and $D S^{*}$, are treated as adjustable parameters. The method is immune from the incorrect understanding of the migration velocity because the current density is not viewed according to the pattern (8). Values of $T S^{*}$ such as +12.5 (in cyclohexane) and -4.17 (in water) were obtained (table 4 in [33]). If the same colloidal particles had been studied by means of thermal field-flow fractionation, values of +8.0 and -12.47 would have been obtained owing to relation (14). In this example, it is clear that the incorrect interpretation of the velocity would cause an important discrepancy between different experimental methods aimed at measuring $S^{*}$. A contrario, we notice that values of $S^{*}$ consistent with those derived from forced Rayleigh scattering have been obtained from thermal-lens measurements [34]. Agreement is achieved because neither method identifies $-D S^{*} \nabla T$ with a migration velocity.

\section{§ 4. Conclusions}

This paper has shown that the measure of partial demixtion $\sigma_{\mathrm{T}} \approx S^{*}$ and the migration velocity $v_{\mathrm{d}}$ are not proportional to each other. They should be conceived of as distinct concepts. $\sigma_{\mathrm{T}}$ refers to the static enrichment of one side relative to the other induced by $\nabla T$, whereas $v_{\mathrm{d}}$ is a dynamic quantity. Their non-proportionality is shown to be due to the inevitable temperature dependence of the diffusivity. What matters in the correction is not the absolute value of $D$, but its relative rate of change with $T$. The correction can be very important if diffusion is 
thermally activated ${ }^{*}$. The reader is also warned about the semantic gap between the notions of transport (entified in $j$ ) and of motion (entified in $d x / d t$ ). They are linked through relation (7).

The conceptual confusion is strengthened in the "non-Brownian approximation" sometimes used in colloidal or micellar systems. That approximation consists in neglecting $D$, or the diffusion current, in a sedimentation experiment when the Péclet number assessing the relative importance of migration over diffusion is large [17, 25]. The limit $D \rightarrow 0$ of a "nonBrownian particle" is often envisaged at a large $R$. However, removing the Fick term in equation (2) suppresses the $d \ln D / d \ln T$ correction in (14) and entails a wrong value of the sign of the migration velocity in an aqueous solution of $\mathrm{NaCl}$ at $0.5 \mathrm{~mol} \cdot \mathrm{L}^{-1}$ at $25^{\circ} \mathrm{C}$. Therefore, the non-Brownian approximation should be avoided in studying thermophoresis. At any rate, a better approximation consists in decomposing $j$ according to equation (8) and removing the total derivative, i.e. the perikinetic contribution to the flow. The latter cannot be dismissed in the steady state, however, as the discussion in $\S 2.2$ has shown. More broadly speaking, because the Nernst-Einstein relation between $D$ and mobility $\mu$ always holds \# and because the response to a force, whether of thermal origin or not, involves the mobility $\mu$, it is hazardous to put $D=0$ while retaining $\mu$ in thermodiffusion studies.

In summary, the view of the Soret coefficient $\sigma_{\mathrm{T}}$, or of the thermophoretic mobility $D S^{*}$, as being proportional to a particle velocity, is erroneous. The error is rooted in the fact that, when a medium is inhomogeneous (owing to a temperature gradient or whatever reason), the coefficient of $n$ in the phenomenological current equation does not have its usual meaning. This can vitiate interpretations of experiment, experimental measurements, theoretical calculations, comparisons of experiment with theory and cross-experimental comparisons. This finding should help to remove discrepancies in the physics of thermophoresis whenever $\left|T \sigma_{\mathrm{T}}\right|$ is not very large.

\section{Acknowledgments}

I am indebted to Alain Bourdon for insightful criticism of the first version of the manuscript. I have benefitted from stimulating conversations with S. Nader Rasuli and Ramin Golestanian.

\section{Appendix A}

To obtain equation (2), we rewrite equation (1) as

$$
\boldsymbol{j}_{1}=-D \nabla n_{1}+D x_{1} \nabla n-D \sigma_{\mathrm{T}} n_{1} \nabla T .
$$

We notice that $n=1 / v$, where $v=v\left(T, p, x_{1}\right)$ is the volume per particle, and

\footnotetext{
* Unpublished data of G. Demouchy and A. Bourdon on ionic-coated maghemite nanoparticules show a $D(T)$ dependence larger than expected from the temperature dependence of the solvent viscosity in the Stokes-Einstein formula with a $T$-independent radius $R$. The thermally activated nature of the weak acid-base equilibrium of the citrate ions coating the nanoparticule surface is suspected to alter the hydrodynamic radius.

\# An activity correction to $D / k T \mu$ is due in a non-ideal solution. See [10], p. 774; or [27], pp. 450-452.
} 


$$
\nabla v=(\partial v / \partial T)_{p, x_{1}} \nabla T+\left(\partial v / \partial x_{1}\right)_{p, T} \nabla x_{1} .
$$

In the right-hand side of (A 2), we recognize the coefficient of thermal expansion $\beta=$ $(1 / v)(\partial v / \partial T)_{p, x_{1}}$ of the solution. Since $\nabla n=-(\nabla v) / v^{2}$, equation (A1) becomes

$$
j_{1}=-D \nabla n_{1}-D x_{1}\left[\beta n \nabla T+\left(\partial v / \partial x_{1}\right)_{p, T}\left(\nabla x_{1}\right) / v^{2}\right]-D \sigma_{\mathrm{T}} n_{1} \nabla T .
$$

The right-hand side of (A 3) contains a $x_{1} \nabla x_{1}$ term which is quadratic in $x_{1}$. Since we are interested in the limit $x_{1} \rightarrow 0$, we drop that term. Similarly, in the limit $x_{1} \rightarrow 0, \beta$ is the coefficient of thermal expansion of the pure solvent. To first order in $n_{1}$ or $x_{1}$, (A 3) yields equation (2).

\section{References}

[1] C. Ludwig, S. B. Akad. Wiss. Wien 20539 (1856).

[2] Ch. Soret, Arch. Sci. Phys. Nat. 248 (1879); C. R. Séances Hebd. Acad. Sci. 91289 (1880); Arch. Sci. Phys. Nat. 4209 (1880).

[3] S. Chapman and F. W. Dootson, Phil. Mag. 33248 (1917).

[4] W. Köhler and S. Wiegand (editors), Thermal Nonequilibrium Phenomena in Fluid Mixtures, Proceedings of the Fourth International Meeting on Thermodiffusion (Springer, Berlin, 2002).

[5] A. Shapiro (editor), Selected Papers from the Fifth International Meeting on Thermodiffusion, Phil. Mag. 83 1965-2219 (2003).

[6] W. S. Broecker, Science 2781582 (1997).

[7] D. Braun and A. Libchaber, Phys. Biol. 1 P1 (2004).

[8] R. Haase, Thermodynamics of Irreversible Processes (Addison-Wesley, Reading, MA, 1969), pp. 357 and 370.

[9] E. L. Cussler, Diffusion: Mass transfer in fluid systems, 2nd edition (Cambridge University Press, Cambridge, MA, 1997), p. 522.

[10] R. Byron Bird, Warren E. Stewart, and Edwin N. Lightfoot, Transport phenomena, 2nd edition (Wiley, New York, 2002), p. 770.

[11] L. D. Landau and E. M. Lifshitz, Fluid Mechanics (Pergamon, Oxford, 1959) (original Russian edition, Mir, Moscow, 1954), section 58.

[12] J. Lenglet, A. Bourdon, J.-C. Bacri and G. Demouchy, Phys. Rev. E 65031408 (2002).

[13] G. S. McNab and A. Meisen, J. Coll. Interf. Sci. 44339 (1973).

[14] J. L. Anderson, Annu. Rev. Fluid Mech. 2161 (1989).

[15] F. Zheng, Adv. Coll. Interf. Sci. 97255 (2002).

[16] E. J. Davis, "Thermophoresis of particles", in Encyclopedia of Surface and Colloid Science, vol. 4 (Marcel Dekker, New York, 2002), pp. 5365-5375.

[17] A. Regazzetti, M. Hoyos and M. Martin, J. Phys. Chem. B 10815285 (2004).

[18] S. Wiegand, J. Phys. Condens. Matter 16 R357 (2004). 
[19] S. Nader Rasuli and Ramin Golestanian, J. Phys. Condens. Matter 17 S1171 (2005).

[20] N. G. van Kampen, J. Phys. Chem. Solids 49673 (1988).

[21] R. Collins and T. Takemori, J. Phys. Condens. Matter 13801 (1989).

[22] N. G. van Kampen, Stochastic Processes in Physics and Chemistry, 2nd edition (NorthHolland, Amsterdam, 1992).

[23] E. Bringuier, Phys. Rev. B 584543 (1998).

[24] P. Lançon, G. Batrouni, L. Lobry and N. Ostrowsky, Europhys. Lett. 5428 (2001).

[25] Theo G. M. Van de Ven, Colloidal Hydrodynamics (Academic, London, 1989).

[26] R. Collins, S. R. Carson and J. A. D. Matthew, Am. J. Phys. 65230 (1997).

[27] W. M. Deen, Analysis of Transport Phenomena (Oxford University Press, New York, 1998), p. 457.

[28] D. R. Caldwell, J. Phys. Chem. 772004 (1973).

[29] Yakov I. Gerasimov (editor), Physical Chemistry, vol. 2 (Mir, Moscow, 1974), p. 409.

[30] S. Iacopini and R. Piazza, Europhys. Lett. 63247 (2003).

[31] M. Martin, C. Van Batten and M. Hoyos, in reference [4], pp. 250-284.

[32] A. C. van Asten, H. F. M. Boelens, W. Th. Kok, P. S. Williams and J. C. Giddings, Sep. Sci. Technol. 29513 (1994).

[33] G. Demouchy, A. Mezulis, A. Bée, D. Talbot, J.-C. Bacri and A. Bourdon, J. Phys. D: Appl. Phys. 371417 (2004).

[34] S. Alves, G. Demouchy, A. Bée, D. Talbot, A. Bourdon and A. M. Figueiredo Neto, in reference [5], pp. 2059-2066.

\section{Figure captions}

Figure 1. A binary fluid mixture is contained in a vessel bounded at $x=0$ and $L$. A temperature gradient $d T / d x<0$ is applied from the top $(x=L)$ to the bottom $(x=0)$.

Figure 2. In the thermophoresis of a finite packet, the temperature-induced gradient of diffusivity $D$ (double arrow) contributes to the drift velocity $v_{\mathrm{d}}$ (simple arrow). 
A binary fluid mixture is contained in a vessel bounded at $x=0$ and $L$. A temperature gradient $\mathrm{dT} / \mathrm{dx}<0$ is applied from the top $(\mathrm{x}=\mathrm{L})$ to the bottom $(\mathrm{x}=0)$.

$1310 \times 1853 \mathrm{~mm}(96 \times 96 \mathrm{DPI})$ 

arrow) contributes to the drift velocity $\mathrm{v}_{\mathrm{d}}$ (simple arrow). 